\title{
Waveguide-integrated van der Waals heterostructure photodetector at telecom wavelengths with high speed and high responsivity
}

\section{Journal Article}

Author(s):

Flöry, Nikolaus; Ma, Ping; Salamin, Yannick (1); Emboras, Alexandros; Taniguchi, Takashi; Watanabe, Kenji; Leuthold, Juerg (1); Novotny, Lukas (i)

Publication date:

2020-07

Permanent link:

https://doi.org/10.3929/ethz-b-000399369

Rights / license:

In Copyright - Non-Commercial Use Permitted

Originally published in:

Nature Nanotechnology 15(2), https://doi.org/10.1038/s41565-019-0602-z

Funding acknowledgement:

165841 - Nonlinear optics with hybrid plasmonic-TMDC materials (SNF) 


\title{
Waveguide-integrated van der Waals heterostructure photodetector at telecom band with high speed and high responsivity
}

\author{
Nikolaus Flöry, ${ }^{1, *}$ Ping Ma, ${ }^{2}, \dagger$ Yannick Salamin, ${ }^{2}$ Alexandros Emboras, ${ }^{2}$ \\ Takashi Taniguchi, ${ }^{3}$ Kenji Watanabe, ${ }^{3}$ Juerg Leuthold, ${ }^{2,} \ddagger$ and Lukas Novotny ${ }^{1,} \S$ \\ ${ }^{1}$ Photonics Laboratory, ETH Zürich, 8093 Zürich, Switzerland \\ ${ }^{2}$ Institute of Electromagnetic Fields, ETH Zürich, 8092 Zürich, Switzerland \\ ${ }^{3}$ National Institute for Material Science, 1-1 Namiki, Tsukuba 305-0044, Japan
}

(Dated: February 19, 2020)

Intensive efforts have been devoted to explore novel optoelectronic devices based on two-dimensional (2D) transition-metal dichalcogenides (TMDCs) owing to their strong lightmatter interaction and distinctive material properties. In particular, photodetectors featuring both high-speed and high-responsivity performance are of great interest for a vast number of applications such as high-data-rate interconnects operated at standardized telecom wavelengths. Yet, the intrinsically small carrier mobilities of TMDCs become a bottleneck for high-speed application use. Here, we present high-performance vertical van der Waals heterostructure-based photodetectors integrated on a silicon photonics platform. Our vertical $\mathrm{MoTe}_{2}$ /graphene heterostructure design minimizes the carrier transit path length in TMDCs and enables a record-high measured bandwidth of at least $24 \mathrm{GHz}$ under a moderate bias voltage of -3 volts. Applying a higher bias or employing thinner $\mathrm{MoTe}_{2}$ flakes boosts the bandwidth even to $50 \mathrm{GHz}$. Simultaneously, our device reaches a high external responsivity of $0.2 \mathrm{~A} / \mathrm{W}$ for incident light at $1300 \mathrm{~nm}$, benefiting from the integrated waveguide design. Our studies shed light on performance trade-offs and present design guidelines for fast and efficient devices. The combination of 2D heterostructures and integrated guided-wave nano photonics defines an attractive platform to realize high-performance optoelectronic devices, such as photodetectors, light-emitting devices and electro-optic modulators.

During the last decade, two-dimensional (2D) materials such as graphene and transition-metal dichalcogenides (TMDCs) [1, 2] have shown great promise for a wide range of photonic and optoelectronic applications [3-8]. $2 \mathrm{D}$ devices have the potential to outperform established and more mature technologies, particularly in terms of form factor, operating conditions and cost-effectiveness.

\footnotetext{
* These authors contributed equally.

$\dagger$ These authors contributed equally.; mapi@ethz.ch

$\ddagger$ leuthold@ethz.ch

$\S$ lnovotny@ethz.ch
}

The possibility to integrate $2 \mathrm{D}$ materials without constraints of crystal lattice matching is disruptive, as it tremendously simplifies manufacturing and increases possible material combinations [9-11]. Graphene, which has been widely used for successful 2D device implementations [12-15], has an intrinsically weak photosensitivity, though its interaction with light can be enhanced using silicon-based integrated photonics, such as optical resonators [16], waveguides [17], or plasmonic structures $[18,19]$. Recently, a plasmonically enhanced telecom band detector device has demonstrated an external responsivity of $\sim 0.5 \mathrm{~A} / \mathrm{W}$ and a fast photoresponse up to at least $110 \mathrm{GHz}$ [18]. While an even higher intrinsic response of $\sim 262 \mathrm{GHz}$ has been predicted for graphenebased devices [20], they suffer from other issues stemming from the gapless nature of graphene, e.g., large dark currents for photodetectors. Alternatively, TMDCs, a semiconducting class of 2D materials, hold great promise for high-performance optoelectronic devices due to their intrinsically strong light-matter interactions [21]. Yet, the integration with a silicon-based platform is challenging, because direct band-to-band transition energies of most TMDCs fall within the absorption band of silicon. Despite of this, few attempts have been made towards the integration of TMDCs with silicon-based structures [22, 23], but high-performance devices, especially operated at standardized telecom bands relevant for applications of information and communication technology (ICT) [24], are still under very limited investigation.

Another major challenge of employing TMDCs for ICT devices is the speed performance. This is of particular importance when TMDCs are employed in photodetectors [25-27]. While impressive photoresponsivities of up to $10^{3} \mathrm{~A} / \mathrm{W}$ and corresponding high photoconductive gains have been demonstrated [28, 29], what is easily overseen is that these high gains typically originate from photogating effects $[26,28]$. The associated long carrier lifetimes inherently limit the speed performance $[26,30]$. Moreover, the carrier mobilities of TMDCs are significantly smaller than those of graphene [23, 31], which poses an obstacle for high-speed device performance. To date, the highest reported bandwidth of TMDC waveguide detectors is below $1 \mathrm{GHz}$ [22, 23], limited by the relatively long transit time of carriers before they are collected. It is therefore highly desirable to investigate TMDC device configurations for an improved speed performance, e.g. comparable to graphene-based devices, 

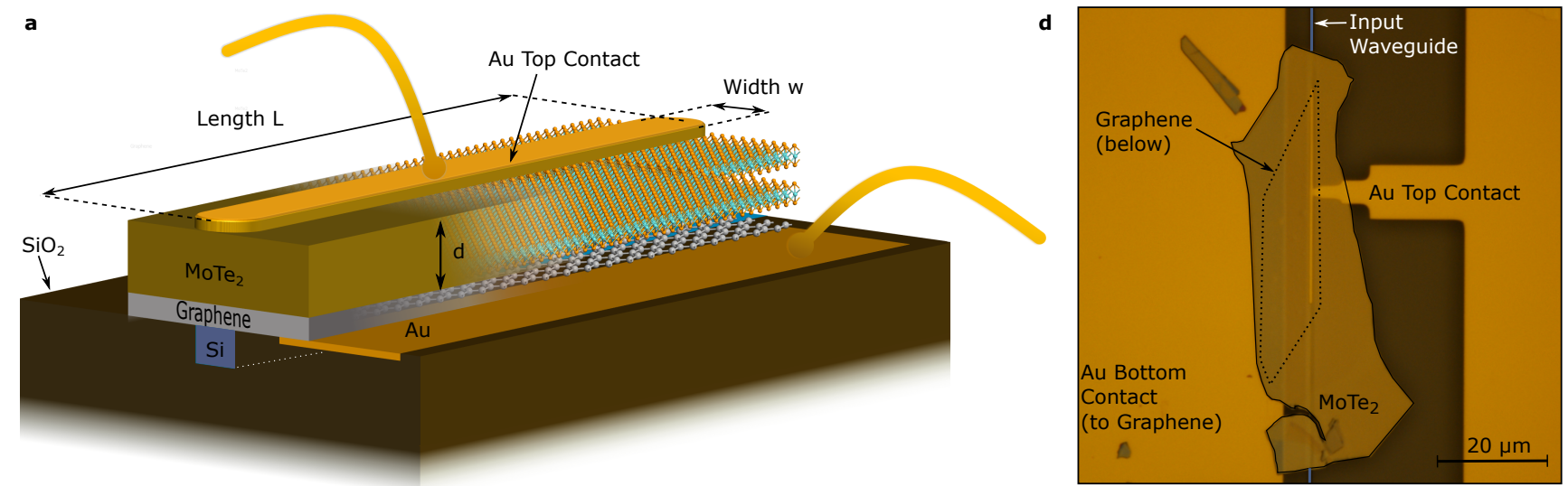

b

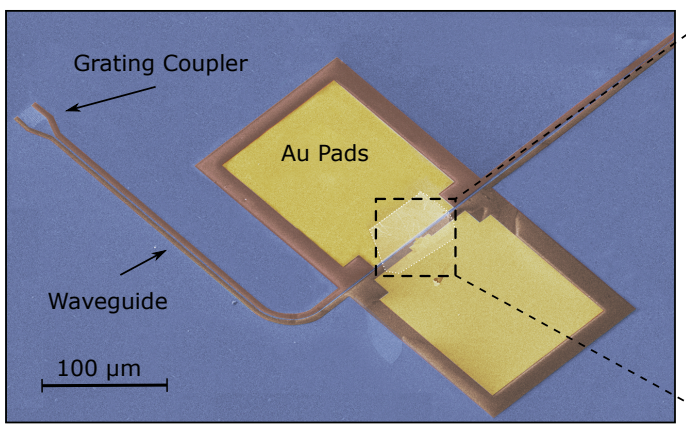

c

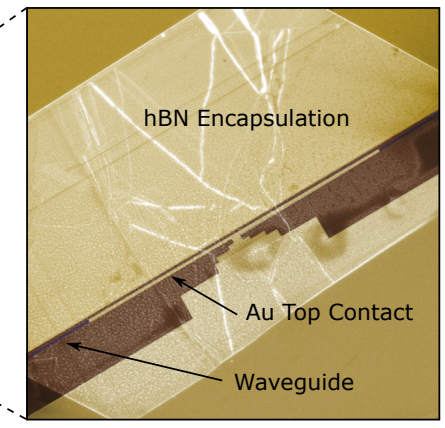

e

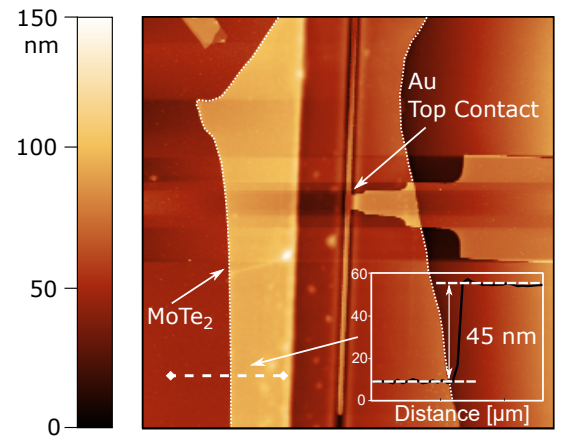

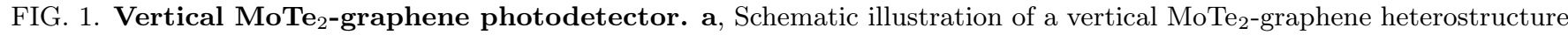
detector coupled to a silicon waveguide buried in $\mathrm{SiO}_{2}$ claddings. Graphene and $\mathrm{MoTe}_{2}$ are connected to gold (Au) bottom and top contacts, respectively. b, False-color scanning electron microscope (SEM) image of a fabricated device, showing the silicon waveguide and grating coupler (GC, both in blue color), the waveguide oxide lateral claddings (in brown color), the metallic structures (in golden color), and the encapsulation hexagonal boron nitride (hBN) layer (in semi-transparent white color). c, Enlarged-view SEM image of the fabricated detector. d, Optical micrograph picture of a fabricated waveguide detector prior to encapsulation with $\mathrm{hBN}$. It shows the graphene flake, the $\mathrm{MoTe}_{2}$ flake, the optical waveguide and metallic structures, including the contact electrode on top of the $\mathrm{MoTe}_{2}$, used for carrier extraction. e, AFM image of the fabricated detector, showing the $\mathrm{MoTe}_{2}$ flake and the metallic contact bar on top of the $\mathrm{MoTe}_{2}$ flake and the planar waveguide. Inset: a cross-sectional line-scan indicating a $\mathrm{MoTe}_{2}$ thickness of $45 \mathrm{~nm}$.

but with higher efficiency.

In this Letter we present high-speed and highresponsivity vertical $\mathrm{MoTe}_{2}$ /graphene van der Waals heterostructure photodetectors integrated with planar silicon photonic waveguides to address the aforementioned key technology challenges. The device design takes full advantage of van der Waals heterostructures as well as of the waveguide integration scheme. Both of these are essential to overcome the intrinsic material constraints.

Figure 1a illustrates the concept of the presented photodetector design. A thin flake of semiconducting fewlayer $\mathrm{MoTe}_{2}$ is introduced as the light absorbing medium. $\mathrm{MoTe}_{2}$ is compatible with silicon integrated photonics, since it exhibits a layer-dependent bandgap and strong light absorption extending into the standard telecom Oband wavelength range $(1260-1360 \mathrm{~nm})[23,32]$. The $\mathrm{MoTe}_{2}$ flake is vertically sandwiched between two parallel electrodes in order to build up a vertical carrier drift path of short distance. The device is operated with transverse electric (TE) polarized light, which has its dominant electric field component parallel to the plane of the $\mathrm{MoTe}_{2}$ flake so as to be efficiently absorbed via band-to-band transitions. Light propagating in the silicon waveguide overlaps evanescently with the absorbing $\mathrm{MoTe}_{2}$ in the active section of the detector. Electron-hole pairs generated by the absorbed photons are efficiently separated and extracted by the uniform electric field applied between the bottom and top electrodes. By design, the collection path of photogenerated carriers is perpendicular to the light propagation direction along the waveguide. As a result, our device has no trade-offs between the carrier transit time limited bandwidth and quantum efficiency.

The high speed of the device is a result of several factors. First, the use of a vertical heterostructure remedies the long transit times of carriers in TMDCs. In previously reported TMDC waveguide detectors, the photo-excited carriers were transported in-plane by lat- 


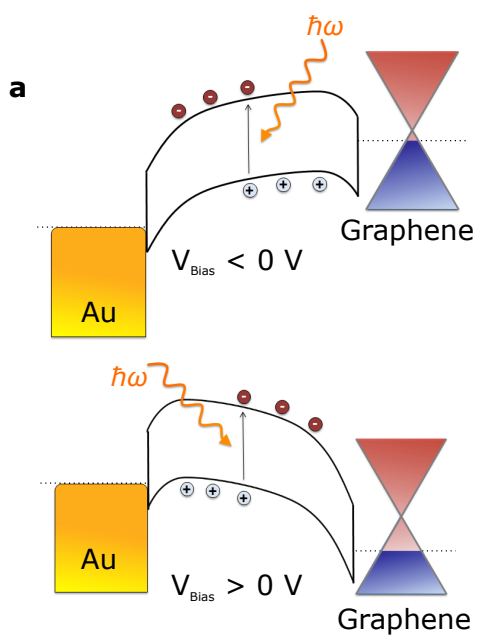

b

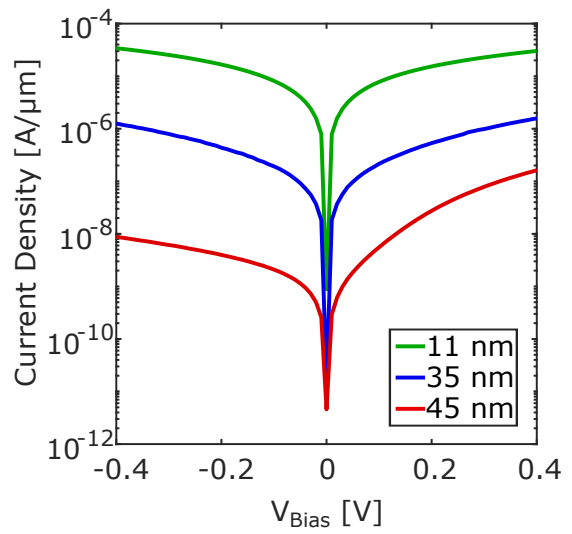

c

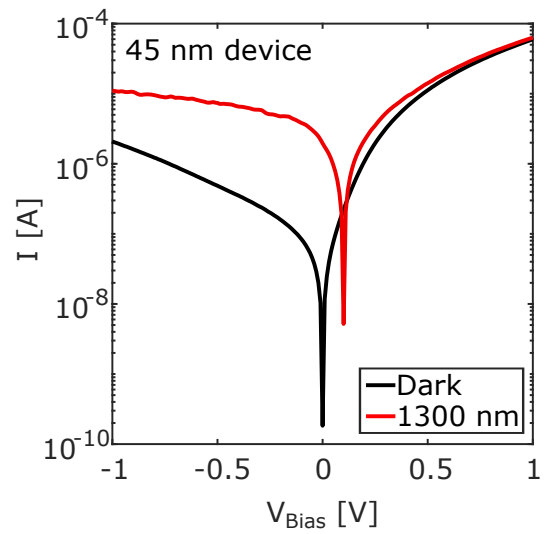

FIG. 2. Electrical Characteristics of the photodetector. a, Schematic illustration of the band diagrams of the vertical $\mathrm{MoTe}_{2}$-graphene heterostructure under negative bias (upper diagram) and positive bias (lower diagram), respectively. b, Dependence of the current density on bias voltage on a logarithmic scale for several devices with different MoTe $\mathrm{M}_{2}$ channel thicknesses (red line, $45 \mathrm{~nm}$; blue line, $35 \mathrm{~nm}$; green line, $11 \mathrm{~nm}$ ). The current density is obtained by normalizing the current to the length of each device. c, Current-voltage (I-V) curves without (black curve) and with (red curve) $1300 \mathrm{~nm}$ light coupled to a detector featuring a $45 \mathrm{~nm}$ thick $\mathrm{MoTe}_{2}$ flake.

eral electric fields over distances of typically a few hundred nanometers, thereby limiting the speed of such devices $[22,23,33]$. A vertical heterostructure on the other hand enables a vertical channel that restricts the transit path length of photoexcited carriers down to a few nanometers, thereby achieving much smaller transit times. Second, monolayer graphene is adopted as a transparent bottom electrode. On one hand, it allows the optical mode to spatially overlap with the $\mathrm{MoTe}_{2}$ absorber and, on the other hand, the high conductivity and carrier mobility of graphene ensures fast carrier extraction and small series resistance. Third, a narrow metallic contact made of thin-film gold ( $\mathrm{Au}$ ) on top of $\mathrm{MoTe}_{2}$, is aligned along the integrated waveguide and creates a small but well-defined vertical carrier extraction channel overlapping with the guided optical mode. The use of such a shaped top electrode allows the active area of the device to be very small, which minimizes the circuital capacitance of the device. Together with the small circuital resistance, this results in a large resistance-capacitance (RC)-limited bandwidth. Besides, the asymmetric contact scheme generates a built-in electric field that contributes to the carrier separation under zero and low bias conditions.

The fabrication process of our devices is elaborated in the Methods and Supplementary Section S1. In short, silicon waveguides were first fabricated using a LOCal Oxidation of Silicon (LOCOS) technique [34, 35] and flakes of $\mathrm{MoTe}_{2}$ and graphene were stacked employing a polymer-based pick-up technique [3, 36]. Figure 1b - e display scanning electron microscope (SEM), optical microscope and atomic force microscope (AFM) images of a fabricated device.

Electrical Characteristics. The electrical behavior of the fabricated devices was characterized first. Figure 2a illustrates the band diagram of the studied heterostructure under positive and negative bias conditions, respectively. Both $\mathrm{MoTe}_{2}$ and graphene are known to be lightly p-doped [37]. The Fermi level of $\mathrm{Au}$ is aligned and pinned close to the valence band of $\mathrm{MoTe}_{2}$ [38-40]. This pinning tends to remain unchanged regardless of the thicknesses of TDMC flakes [38]. Graphene on the other hand forms a smaller and tunable Schottky barrier after contacting $\mathrm{MoTe}_{2}[29,30]$. The difference in work function of $\mathrm{Au}$ and graphene leads to a built-in potential in the device. Applying a bias voltage increases the potential drop across the device that drives the photoexcited carriers. Figure 2b shows the current density against the applied bias voltage for devices comprising flakes of three different $\mathrm{MoTe}_{2}$ thicknesses and lengths, more exactly, $11 \mathrm{~nm}$ and $20 \mu \mathrm{m}, 35 \mathrm{~nm}$ and $33 \mu \mathrm{m}$, and $45 \mathrm{~nm}$ and $40 \mu \mathrm{m}$. The measured current is normalized to the effective detector length of each device to account for the size-variations of the used flakes. As expected, the highest current density can be observed for the thinnest device, as a result of the short resistive $\mathrm{MoTe}_{2}$ channel. Moreover, asymmetric current densities under positive and negative bias conditions, induced by the asymmetric contacting scheme, are visible and more pronounced for thicker devices, most likely originating from a $\mathrm{MoTe}_{2}$ thickness-dependent Schottky barrier height between graphene and $\mathrm{MoTe}_{2}$, as previously studied and reported in graphene-TMDC heterostructures $[11,30]$. This asymmetric contacting scheme also 


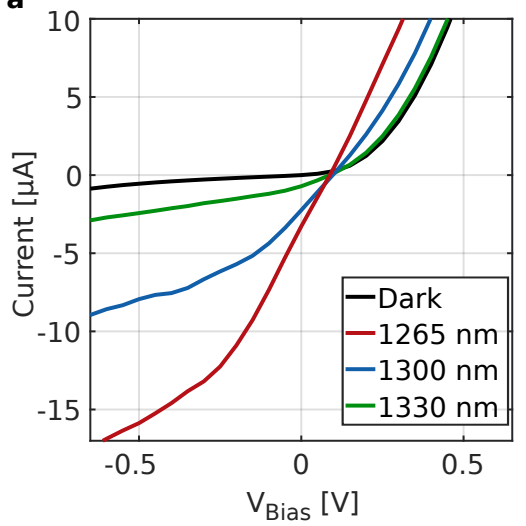

b

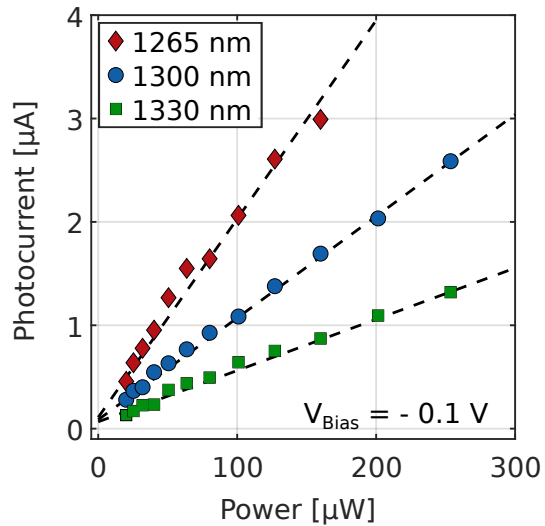

c

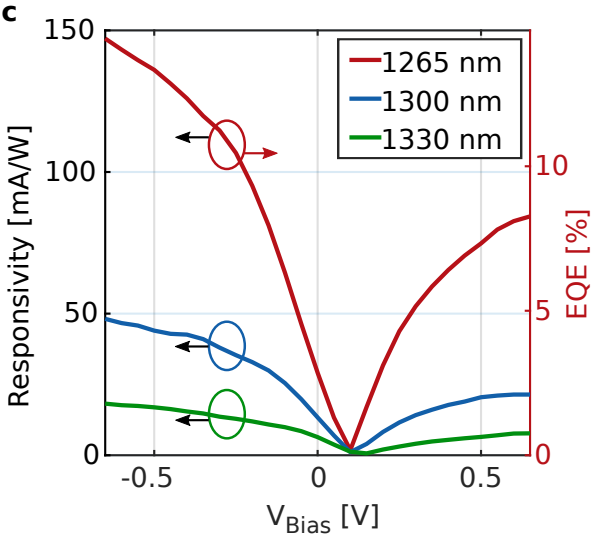

d

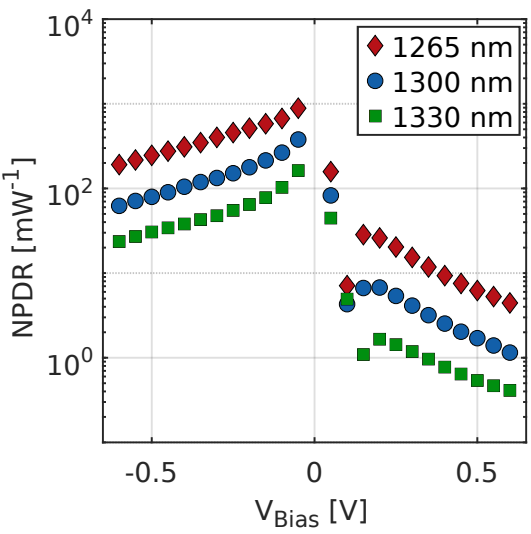

e

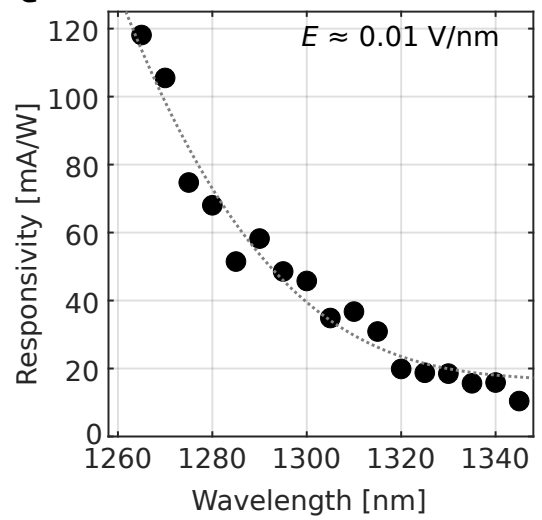

$\mathbf{f}$

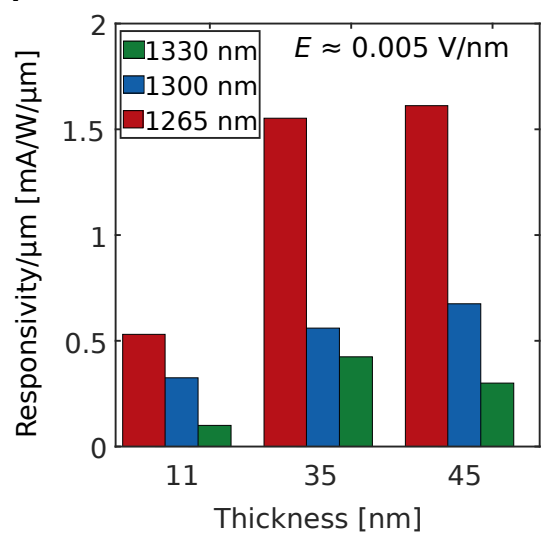

FIG. 3. Steady-state photoresponse of a waveguide photodetector featuring a MoTe 2 thickness of 45 nm. a, Current-voltage (I-V) curves with and without light for three different wavelengths (black, dark current; red, 1265 nm; blue, $1300 \mathrm{~nm}$; green, $1330 \mathrm{~nm}$ ). The optical power is $150 \mu \mathrm{W}$ for all three wavelengths. $\mathbf{b}$, Measured photocurrent as a function of the optical power at $-0.1 \mathrm{~V}$ bias. c, Measured responsivity (left vertical axis in black) as a function of the applied bias voltage for three different wavelengths. Derived external quantum efficiency (EQE, right vertical axis in red) for input light at $1265 \mathrm{~nm}$. A responsivity of $150 \mathrm{~mA} / \mathrm{W}$ and a EQE of $14 \%$ are obtained at a low bias voltage of $-0.6 \mathrm{~V}$ and for input light at $1265 \mathrm{~nm}$. d, Normalized photocurrent-to-dark-current ratio (NPDR) as a function of the applied bias voltage. The highest NPDR is calculated to be $1000 \mathrm{~mW}^{-1}$ for input light at $1265 \mathrm{~nm}$ and small bias conditions. e, External responsivity as a function of wavelength for $-0.5 \mathrm{~V}$ bias. The wavelength dependent photoresponse agrees with the absorption spectrum of MoTe ${ }_{2}$. Dots are data and the dashed line is a guide to the eye. f, Comparison of the responsivity normalized by the device lengths for devices with different flake thicknesses and identical electric fields $E \sim 0.005 \mathrm{~V} / \mathrm{nm}$. Red, blue, and green bars are for light at $1265 \mathrm{~nm}, 1300 \mathrm{~nm}$ and $1330 \mathrm{~nm}$, respectively.

manifests itself in a pronounced photoresponse at zero bias $\left(\mathrm{V}_{\text {Bias }}=0 \mathrm{~V}\right)$ resulting from an intrinsic built-in field. Figure 2c shows current-voltage (I-V) curves of the $45 \mathrm{~nm}$ thick device with and without $1300 \mathrm{~nm}$ light coupled into the waveguide. Without any applied voltage the device is already capable to efficiently separate photo-excited electron-hole pairs and to generate a considerable photocurrent of $2 \mu \mathrm{A}$ for $150 \mu \mathrm{W}$ input power, with negligible dark current.

Steady-state photoresponse. The steady-state photoresponse of the fabricated devices was evaluated using linearly TE-polarized laser light with center wavelength of $1300 \mathrm{~nm}$ coupled into the integrated waveguides via GCs. Figure 3a shows I-V measurements with and without light coupled into a device with a $45 \mathrm{~nm}$ thick $\mathrm{MoTe}_{2}$ flake. Unless otherwise specified, all data presented in Fig. 3 are based on this specific device. A pronounced increase in current, especially under negative bias voltages, is measured when light is coupled in. The power dependence of this photocurrent is shown in Fig. 3b for different wavelengths. A linear dependence is observed within the measured power range. The photoresponsivity is extracted as the ratio of the photocurrent and the incident optical power delivered to the photodetector. It increases with the applied bias voltage that assists the extraction of carriers. Increasing the applied bias to moderate values of up to -0.6 $\mathrm{V}$, as shown in Fig. 3c, yields photoresponsivities of 
$150 \mathrm{~mA} / \mathrm{W}, 50 \mathrm{~mA} / \mathrm{W}$ and $20 \mathrm{~mA} / \mathrm{W}$ for wavelengths of $1265 \mathrm{~nm}, 1300 \mathrm{~nm}$ and $1330 \mathrm{~nm}$, respectively. This corresponds to an external quantum efficiency (EQE) $\eta_{E Q E}$ of $14 \%$ at $1265 \mathrm{~nm}\left(\eta_{E Q E}=R \cdot \hbar \omega / q, R\right.$ denoting the responsivity, $\hbar$ the reduced Planck constant, $\omega$ the light angular frequency, and $q$ the elementary charge). We intentionally keep the bias voltage low in steady-state photodetection measurements in order not to damage the devices. In fact, the photocurrent and the EQE are expected to further increase for larger applied bias voltages, limited only by break down and saturation of absorption (see Supplementary Section S3,S4 for details on the limits of the responsivity).

Normalized photo-dark-current ratio (NPDR) [41] is another important performance indicator of a photodetector. As shown in Fig. 3d, the NPDR of our devices is in the order of $100 \mathrm{~mW}^{-1}$ under negative bias conditions and approaches $1000 \mathrm{~mW}^{-1}$ for shorter wavelengths and small bias voltages. This performance outperforms graphene-based photodetectors $[14,42]$ by almost an order of magnitude. The dark current can be further reduced by enhancing the contact asymmetry [43], applying gating [44] or inserting a thin charge blocking layer with a wide bandgap between $\mathrm{MoTe}_{2}$ and one of the electrodes $[45,46]$ (see Supplementary Section S5 for details). We further characterized the dependence of the photoresponse on the wavelength of the incoupled light. As shown in Fig. 3e, the measured responsivity spectrum agrees with the wavelength dependent absorption of the few-layer $\mathrm{MoTe}_{2}$ flake. It exhibits stronger absorption and hence a higher photoresponsivity for shorter wavelengths.

In Fig. 3f we compare devices with different $\mathrm{MoTe}_{2}$ thicknesses. The figure shows the responsivities, normalized to device lengths, for light with wavelengths of $1265 \mathrm{~nm}, 1300 \mathrm{~nm}$ and $1330 \mathrm{~nm}$. The observed trend clearly shows that devices consisting of thicker $\mathrm{MoTe}_{2}$ flakes possess a higher photoresponse, which is due to the higher absorption in the thicker semiconducting channel. The behaviour for $1265 \mathrm{~nm}$ (red bars) is the same as for $1300 \mathrm{~nm}$ (blue bars) and $1330 \mathrm{~nm}$ (green bars), despite the overall lower responsivity. These characteristics, together with the observed linear power dependence and the wavelength dependent photocurrent, indicate that the observed photoresponse stems from photo-excited carriers generated by light absorption in the semiconducting $\mathrm{MoTe}_{2}$. This is further verified by an negligible photoresponsivity at even longer wavelengths of $1575 \mathrm{~nm}$ below the bandgap of $\mathrm{MoTe}_{2}$, thereby ruling out other effects (see Supplementary Section S6).

High-frequency photoresponse. To characterize the speed performance of the devices we used the experimental setup illustrated in Fig. 4a (see Methods for detailed description). Figure $4 \mathrm{~b}$ shows the measured frequency response of three photodetector devices with different $\mathrm{MoTe}_{2}$ flake thicknesses. In all three cases the response stays flat from $100 \mathrm{kHz}$ to $\mathrm{GHz}$ frequencies and then drop off. A standard low pass filter model was used to fit the data, revealing the $3 \mathrm{~dB}$ roll-off frequency of each measurement. It is evident from Fig. $4 \mathrm{~b}$ that devices with thinner $\mathrm{MoTe}_{2}$ exhibit a faster photoresponse. For the $45 \mathrm{~nm}$ and $35 \mathrm{~nm}$ thick devices we measure a roll-off frequency of $12 \mathrm{GHz}$ at $-3.5 \mathrm{~V}$ and $24 \mathrm{GHz}$ at $-3 \mathrm{~V}$ bias, respectively. Whereas the bandwidth of the $11 \mathrm{~nm}$ thick $\mathrm{MoTe}_{2}$ device exceeds $30 \mathrm{GHz}$ already at a low bias of $-0.4 \mathrm{~V}$, which is beyond the bandwidth of the instruments used in the experiment. The extrapolated $3 \mathrm{~dB}$ roll-off frequency is nearly $50 \mathrm{GHz}$. To the best of our knowledge, this is the highest reported bandwidth of a TMDC-based photodetector, outperforming those of previous studies by more than one order of magnitude $[22,23]$. For the $35 \mathrm{~nm}$ thick $\mathrm{MoTe}_{2}$ device we show in Fig. 4c the influence of the bias voltage on the frequency response in the roll-off regime. The $3 \mathrm{~dB}$ roll-off frequency increases with the applied bias since photo-excited carriers are separated faster by large electric fields. Figure $4 \mathrm{~d}$ plots the roll-off frequencies as a function of the bias voltage for all three devices. It reveals that the bandwidth increases monotonously with bias voltage. This is because the velocity of the carriers increases linearly with applied bias. Furthermore, a high bias not only increases the bandwidth but also leads to an enhanced photoresponsivity, as a result of the reduced carrier recombination. As shown in Fig. 4e, the photoresponsivity increases with the bias voltage, approaching $200 \mathrm{~mA} / \mathrm{W}$ at a bias of -3 volt for incident light at $1300 \mathrm{~nm}$ for the $35 \mathrm{~nm}$ thick $\mathrm{MoTe}_{2}$ device, corresponding to an EQE close to $20 \%$.

It is meaningful to determine the circuital characteristics of the devices, as the RC products could also impose limits on the time response. To this end, we experimentally characterized the alternating current (AC) capacitances of the studied devices (see Supplementary Section S7). Thanks to the compact size of the devices, the measured capacitances are significantly smaller than those of typical top illuminated devices [47] and are in the range of a few tenths of femtofarads. Thus, the RC limited bandwidths are larger than $100 \mathrm{GHz}$ if the devices are connected to a $50 \mathrm{Ohm}$ load. We therefore conclude that the bandwidths of our graphene-MoTe ${ }_{2}$ photodetectors are mainly limited by the carrier dynamics (see Supplementary Section S8).

Discussion. As discussed in Fig. 4d, the roll-off frequencies depend on the bias voltage. Moreover, for a constant electric field, thin devices show higher roll-off frequencies, as shown in Fig. 5a. These characteristics disclose drift-diffusive transport to govern the charge carrier dynamics in our devices. On average, the photoexcited carriers need to drift over a length $d / 2$, where $d$ is the vertical distance between electrodes, i.e. the flake thickness of $\mathrm{MoTe}_{2}$. The velocity of carriers is given by $\nu=\mu E \approx \mu V / d$, where $\mu$ is the out-of-plane mobility of carriers, $E$ the electric field across the device and $V$ the applied bias voltage. The transit time for carriers to be collected can be derived as $\tau_{t r}=d^{2} / 2 \mu V$ and the corresponding transit time limited frequency, $f_{3 d B}$, 

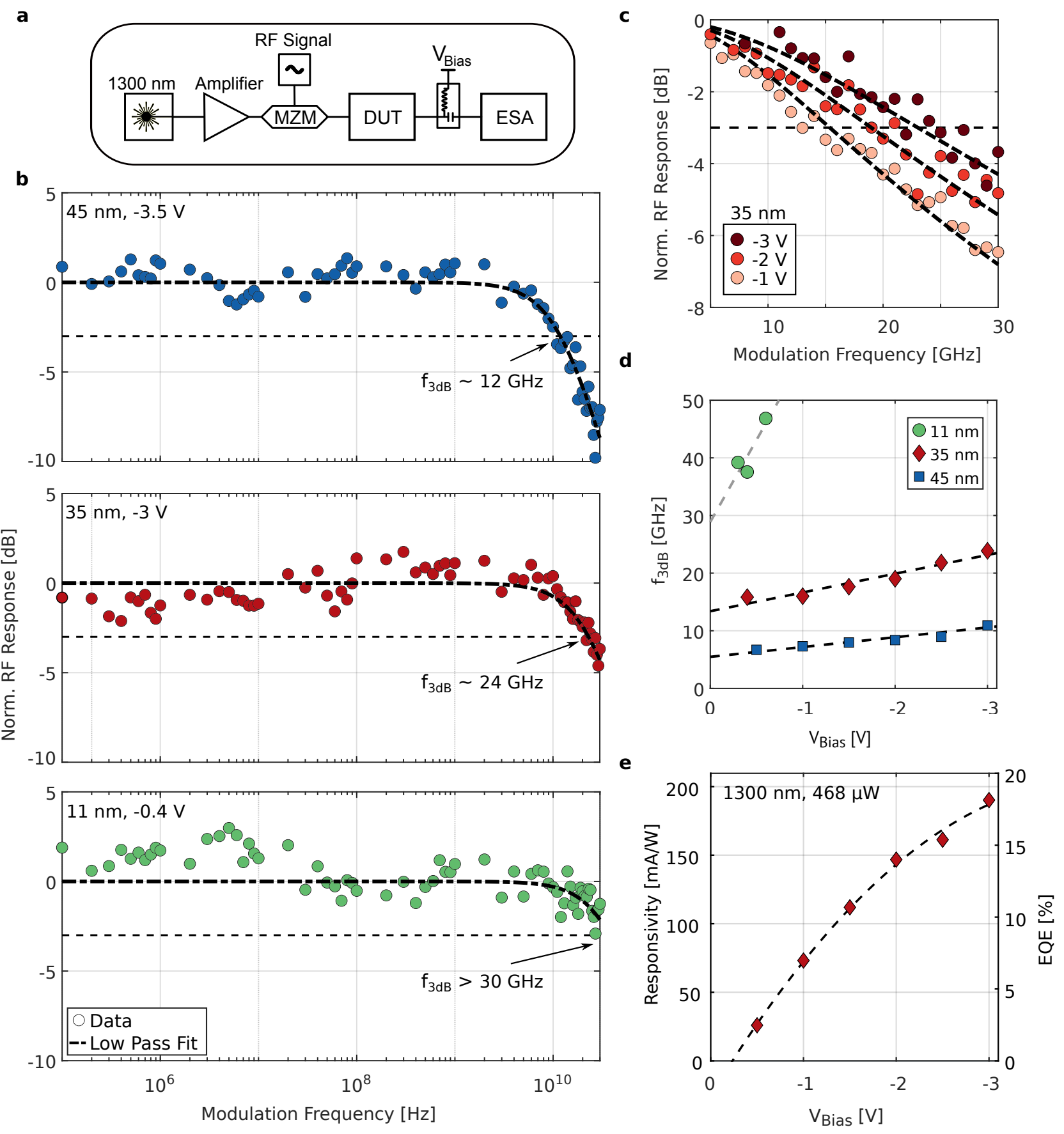

FIG. 4. Dynamic characterization of vertical photodetectors. a, Frequency response measurement setup. MZM, MachZehnder modulator; DUT, device under test; ESA, electrical spectrum analyzer. b, Normalized radio frequency (RF) signal response as a function of the modulation frequency of the input signal for three devices. A single pole low-pass filter is used to fit the data points and to extract the $3 \mathrm{~dB}$ roll-off frequency $f_{3 d B}$ for each device. The thicknesses of the MoTe $\mathrm{e}_{2}$ flakes and the applied bias voltages are indicated in the figures. c, Frequency response of the $35 \mathrm{~nm}$ thick $\mathrm{MoTe}_{2}$ device for different bias voltages. $\mathbf{d}$, Dependence of the roll-off frequency $f_{3 d B}$ on the applied bias voltage and for different MoTe $\mathrm{Mlake}_{2}$ thicknesses (blue scatters, $45 \mathrm{~nm}$; red rhombuses, $35 \mathrm{~nm}$; green dots, $11 \mathrm{~nm}$ ). The linear trend indicates that the bandwidth is transit time limited. e, Simultaneously measured responsivity and corresponding external quantum efficiency as a function of the applied bias voltage for input light at $1300 \mathrm{~nm}$ with power intensity of $468 \mu \mathrm{W}$.

is shown as the black dotted line in Fig. 5b. This sim- ple model can be used to fit the slope of the linear be- 

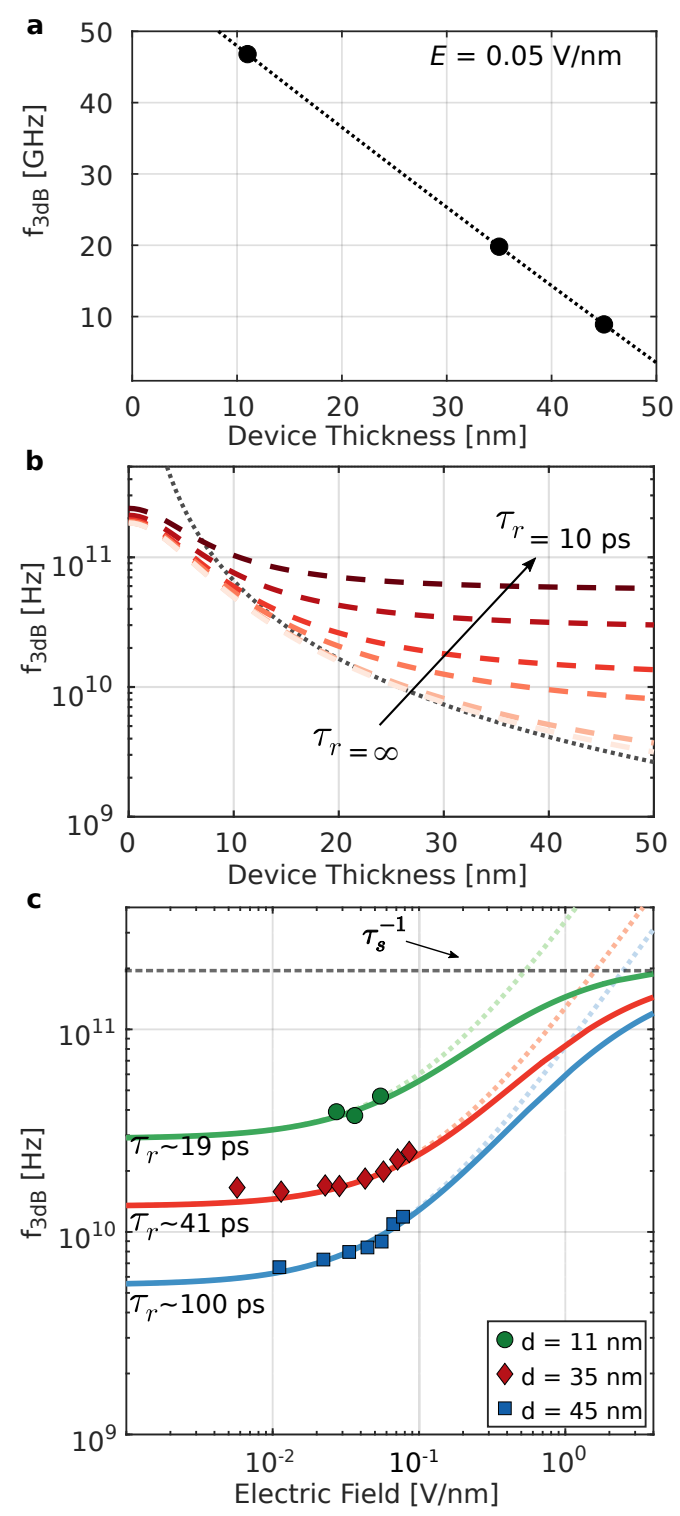

FIG. 5. Comparison of devices with different $\mathrm{MoTe}_{2}$ thicknesses and under different bias conditions. a, Roll-off frequencies $f_{3 d B}$ for different $\mathrm{MoTe}_{2}$ thicknesses $d$ but at identical electric field $E$. b. Predicted roll-off frequencies $f_{3 d B}$ for different $\mathrm{MoTe}_{2}$ thicknesses. The dotted line is a plot based on a simple transit time model. The colorful dashed lines are plots based on the adapted transit time model taking into account the carrier recombination channel for devices under different bias conditions. c, Measured rolloff frequencies $f_{3 d B}$ versus applied electrical fields $\left(\mathrm{V}_{\text {Bias }} / d\right)$ for different $\mathrm{MoTe}_{2}$ thicknesses. The indicated fitting parameters $\tau_{r}$ correspond to the recombination lifetimes, which are $\mathrm{MoTe}_{2}$ thickness dependent.

havior found in Fig. 4d, providing an estimate for the out-of-plane mobility of $0.03 \mathrm{~cm}^{2} \mathrm{~V}^{-1} \mathrm{~s}^{-1}$ for all three devices, in good agreement with values reported in the literature $[11,47]$. In order to explain the different intercepts of the linear fits at zero bias in Fig. 4d, however, an ad- ditional channel needs to be considered. As investigated recently by Massicote et al. who employed time-resolved pump-probe measurements to study the carrier dynamics of $\mathrm{WSe}_{2}$ heterostructures, there exists a loss mechanism stemming from carrier recombination [47]. This gives rise to a lower bound of the bandwidth at small bias voltages and the total extraction time $\tau$ can be described by $\tau^{-1}=\tau_{t r}^{-1}+\tau_{r}^{-1}$, where $\tau_{r}$ denotes the carrier recombination lifetime.

Figure 5b illustrates the impact of this additional parallel channel on the carrier dynamics. Treating the recombination lifetime $\tau_{r}$ as a variable, the thicknessdependent roll-off frequencies for various lifetime values are plotted. While in a purely transit time limited case the theoretical maximal roll-off frequency falls off quickly with increasing thickness $d$ (black dotted line), taking the lifetime $\tau_{r}$ into account significantly flattens out this drop-off (colored dashed lines). This behavior is more remarkable if the carrier recombination lifetime $\tau_{r}$ is small, thereby enabling higher roll-off frequencies. In contrast, a slow recombination (large $\tau_{r}$ ) slows down the overall carrier dynamics.

For completeness, an additional timescale has to be taken into account when looking at the upper bound of the theoretical bandwidth, namely the lifetime of interfacial processes describing the actual transfer of carriers from the semiconducting channel to the electrodes, denoted as $\tau_{s}$ in Fig. 5b. While the recombination lifetime $\tau_{r}$ acts as a parallel loss channel, $\tau_{s}$ is a process in series with the photo-carriers extraction. Therefore, it starts to play a role when the transit time reaches a few picoseconds for very thin devices or for devices under very strong electric fields. The lifetime of such a process was reported to be thickness-independent and on the order of $2-5$ ps [48]. This is beyond our experimental study, but shows that the roll-off frequencies may converge to an upper limit beyond $200 \mathrm{GHz}$. This predicted ultimate performance is comparable to the estimated intrinsic bandwidth of graphene, which is about $260 \mathrm{GHz}$ [20]. We can now compare the extracted roll-off frequencies of different devices as a function of electric field $E$ with the modeled roll-off frequencies using $f_{3 d B}=0.55 / \tau[47,49,50]$. The complete carrier rate equation is given by:

$$
\frac{1}{\tau}=\frac{1}{\tau_{s}+\frac{d}{2 \mu E}}+\frac{1}{\tau_{r}}
$$

We find a good agreement between our experimental results and the fit. As expected, thinner channels result in higher roll-off frequencies when applying the same field across the device. A stronger field raises the roll-off frequency resulting from a shorter transit time of the accelerated photo-excited carriers. This dependence is more pronounced at high fields and less efficient at low fields, for which the roll-off frequencies start to plateau. Using $\tau_{r}$ as a fitting parameter we obtain a clear dependence on the thickness of the $\mathrm{MoTe}_{2}$ flakes. We find the recombination lifetimes to be relatively small $\left(\tau_{r}=19 \mathrm{ps}\right)$ for thin devices $(d=11 \mathrm{~nm})$ and large $\left(\tau_{r}=100 \mathrm{ps}\right)$ 
for thick devices $(d=45 \mathrm{~nm})$. This trend has been observed previously in transient absorption studies of other TMDCs [51, 52]. The observation that $\tau_{r}$ scales with the thickness $d$ can be attributed to surface defects. Because thin TMDC flakes have a high surface-to-volume ratio, they are more susceptible to surface defects. Thus, thin flakes are more favorable for fast carrier dynamics, firstly due to their short carrier transit channel and secondly due to their intrinsically short recombination lifetimes. On the other hand, when the TMDC flake gets even thinner (e.g., $d<10 \mathrm{~nm}$ ), the reciprocally increased capacitance may eventually limit the device bandwidth. Our analysis reveals that an optimum thickness around $10 \mathrm{~nm}$ offers the highest bandwidth for a resistively loaded device (see Supplementary Section S8).

Furthermore, since the extraction rate $\Gamma=1 / \tau_{t r}$ increases with thickness, the internal quantum efficiency (IQE), given as the ratio of extracted carriers and total photo-generated carriers, is expected to increase for thin devices. However, as $\tau_{r}$ is small for thin devices, there is also a fast recombination channel that counteracts the carrier extraction. As a result, we find the IQE to be nearly equal (up to $\sim 40 \%$ for our measured voltage range) for different thicknesses, and only dependent on the applied field (more details in Supplementary Section S3). The reason why a thin device typically exhibits a lower responsivity or a lower EQE than those of thicker devices, can be attributed to the reduced photoactive material. Hence, it appears that a low responsivity is the price to pay for a high bandwidth. However, the responsivity of our waveguide-integrated devices can readily be improved by simply increasing the length of the flake on the waveguide. Thus, our proposed design concept shows a viable scheme to overcome the common tradeoff between high (external) efficiency and fast intrinsic photoresponse.

In conclusion, our results have profound impact on the understanding and development of practical TMDC optoelectronic devices. The presented waveguide coupled vertical heterostructure device concept paves a way to boost the speed performance of TMDC-based photodetectors to the same order of magnitude as those of e.g. graphene, while offering advantages in terms of lightmatter interaction and dark currents (a comparison given in Supplementary Section S9). With record-high directly measured bandwidths, our results extend the potential of TMDC materials for practical optoelectronic devices particularly in the fields of high-speed applications such as high-data-rate optical interconnects operated at standard telecom wavelengths. As the integration of $2 \mathrm{D}$ materials is platform-independent, our developments and findings are not limited to silicon photonics, but could also be used to explore and advance devices based on other technologies, such as silicon nitride $[53,54]$ or flexible substrate platforms [55].

\section{METHODS}

Device fabrication. Photodetectors were fabricated on standard silicon-on-insulator (SOI) wafers. Buried silicon waveguides with dimensions of an effective width $w=400 \mathrm{~nm}$ and a height $h=220 \mathrm{~nm}$ were first built by using the LOCal Oxidation of Silicon (LOCOS) technique (see Supplementary Information, S2). Grating couplers (GCs) were produced by a shallow etching of silicon. A top $5 \mathrm{~nm}$ thick SiN dielectric layer was then deposited by atomic layer deposition for an electrical isolation from the silicon layer underneath. Next to the waveguide, bottom metallic pads, which were used to contact the graphene electrode, were defined by electron-beam lithography and evaporation of $5 \mathrm{~nm} \mathrm{Ti}$ and $50 \mathrm{~nm} \mathrm{Au}$. Mechanical exfoliation was employed to obtain crystalline flakes of $\mathrm{MoTe}_{2}$, graphene and hexagonal boron nitride (hBN), which were identified with an optical microscope and whose thicknesses were characterized with an AFM. The graphene $\mathrm{MoTe}_{2}$ heterostructure were stacked by using a polymer-based pick-up technique with a polydimethylsiloxane (PDMS) polypropylene carbonate (PPC) stamp, transferred to the device chips, and aligned to the silicon waveguides with the help of the micromechanical stage of a SUSS MJB4 mask aligner. $200 \mathrm{~nm}$ wide and $20 \mathrm{~nm}$ thick top $\mathrm{Au}$ contact pads were formed again by electron-beam lithography, metal evaporation, and a lift-off process. The whole devices were finally encapsulated by $\mathrm{hBN}$ flakes. The measurements were performed at ambient conditions at room temperature.

Electrical and optical measurements. Electrical characterization was performed using a pico-ampere precision source to apply biases and read out the current. The metallic contact pads connected to the bottom graphene electrodes were biased positively or negatively, while the top contact was grounded. The steady-state photoresponse was evaluated using linearly TE-polarized laser light with center wavelength of $1300 \mathrm{~nm}$. Light was coupled into the integrated waveguides via GCs. The coupling loss of a GC was characterized with the help of nearby reference structures on the same chip (see Supplementary Section S2 for details). The high-frequency measurements were done on a separate experimental setup. An optical intensity Mach-Zehnder modulator (MZM, $\mathrm{u}^{2} \mathrm{t}$ MZMO2120) with $30 \mathrm{GHz}$ electro-optic bandwidth was driven by a radio frequency (RF) signal from an electrical synthesizer and used to modulate an amplified continuous-wave laser tunable around $1300 \mathrm{~nm}$. The modulated laser light was coupled into the device via GCs. A bias-tee was used to apply a direct current (DC) bias to the devices. The generated RF electrical signals were extracted from the devices with a high-speed microwave probe and measured with an electrical spectrum analyzer (ESA). The frequency responses ranging from $100 \mathrm{kHz}$ to $30 \mathrm{GHz}$ were measured under various bias voltages. The whole measurement setup was calibrated 
request. using a commercially available high-speed photodetector

with a bandwidth of $72 \mathrm{GHz}\left(\mathrm{u}^{2} \mathrm{t}\right.$ XPDV3120R).

\section{Data Availability}

The data that support the findings of this study are available from the corresponding author upon reasonable
[1] Wang, Q. H., Kalantar-Zadeh, K., Kis, A., Coleman, J. N. \& Strano, M. S. Electronics and optoelectronics of two-dimensional transition metal dichalcogenides. Nat. Nanotechnol. 7, 699-712 (2012).

[2] Xiao, J., Zhao, M., Wang, Y. \& Zhang, X. Excitons in atomically thin 2D semiconductors and their applications. Nanophotonics 6, 1309-1328 (2017).

[3] Britnell, L. et al. Strong light-matter interactions in heterostructures of atomically thin films. Science 340, 13111314 (2013).

[4] Withers, F. et al. Light-emitting diodes by bandstructure engineering in van der Waals heterostructures. Nat. Mater. 14, 301-306 (2015).

[5] Sun, Z., Martinez, A. \& Wang, F. Optical modulators with 2D layered materials. Nat. Photon. 10, 227-238 (2016).

[6] Bonaccorso, F., Sun, Z., Hasan, T. \& Ferrari, A. C. Graphene photonics and optoelectronics. Nat. Photon. 4, 611-622 (2010).

[7] Boltasseva, A. \& Shalaev, V. M. Transdimensional photonics. ACS Photonics 6, 1-3 (2019).

[8] Manzeli, S., Ovchinnikov, D., Pasquier, D., Yazyev, O. V. \& Kis, A. 2D transition metal dichalcogenides. Nat. Rev. Mater. 2, 17033 (2017).

[9] Mueller, T., Pospischil, A. \& Furchi, M. M. 2D materials and heterostructures for applications in optoelectronics. Proc. SPIE 9467, Micro- and Nanotechnology Sensors, Systems, and Applications VII 9467, 946713 (2015).

[10] Liu, Y. et al. Van der Waals heterostructures and devices. Nat. Rev. Mater. 1, 16042 (2016).

[11] Yu, W. J. et al. Vertically stacked multi-heterostructures of layered materials for logic transistors and complementary inverters. Nat. Mater. 12, 246-252 (2013).

[12] Kim, K., Choi, J. Y., Kim, T., Cho, S. H. \& Chung, H. J. A role for graphene in silicon-based semiconductor devices. Nature 479, 338-344 (2011).

[13] Schuler, S. et al. Graphene photodetector integrated on a photonic crystal defect waveguide. ACS Photonics 5, 4758-4763 (2018).

[14] Gan, X. et al. Chip-integrated ultrafast graphene photodetector with high responsivity. Nat. Photon. 7, 883887 (2013).

[15] Hone, J. et al. High-responsivity graphene-boron nitride photodetector and autocorrelator in a silicon photonic integrated circuit. Nano Lett. 15, 7288-7293 (2015).

[16] Phare, C. T., Daniel Lee, Y. H., Cardenas, J. \& Lipson, M. Graphene electro-optic modulator with $30 \mathrm{GHz}$ bandwidth. Nat. Photon. 9, 511-514 (2015).

[17] Schall, D. et al. Record high bandwidth integrated graphene photodetectors for communication beyond 180 gb/s. Optical Fiber Communication Conference, M2I.4
(2018).

[18] Ma, P. et al. Plasmonically enhanced graphene photodetector featuring $100 \mathrm{Gbit} / \mathrm{s}$ data reception, high responsivity, and compact size. ACS Photonics 6, 154-161 (2019).

[19] Ding, Y. et al. Ultra-compact integrated graphene plasmonic photodetector with bandwidth above 110 ghz. Nanophotonics 0 (2019).

[20] Urich, A., Unterrainer, K. \& Mueller, T. Intrinsic response time of graphene photodetectors. Nano Lett. 11, 2804-2808 (2011).

[21] Mak, K. F. \& Shan, J. Photonics and optoelectronics of 2D semiconductor transition metal dichalcogenides. Nat. Photon. 10, 216-226 (2016).

[22] Bie, Y.-Q. Q. et al. A MoTe 2 -based light-emitting diode and photodetector for silicon photonic integrated circuits. Nat. Nanotechnol. 12, 1124-1129 (2017).

[23] Ma, P. et al. Fast $\mathrm{MoTe}_{2}$ waveguide photodetector with high sensitivity at telecommunication wavelengths. $A C S$ Photonics 5, 1846-1852 (2018).

[24] Ferrari, A. C. et al. Graphene-based integrated photonics for next-generation datacom and telecom. Nat. Rev. Mater. 3, 392-414 (2018).

[25] Koppens, F. H. L. et al. Photodetectors based on graphene, other two-dimensional materials and hybrid systems. Nat. Nanotechnol. 9, 780-793 (2014).

[26] Konstantatos, G. Current status and technological prospect of photodetectors based on two-dimensional materials. Nat. Comm. 9, 5266 (2018).

[27] Buscema, M. et al. Photocurrent generation with twodimensional van der Waals semiconductors. Chem. Soc. Rev. 44, 3691-3718 (2015).

[28] Lopez-Sanchez, O., Lembke, D., Kayci, M., Radenovic, A. \& Kis, A. Ultrasensitive photodetectors based on monolayer $\mathrm{MoS}_{2}$. Nat. Nanotechnol. 8, 497-501 (2013).

[29] $\mathrm{Yu}, \mathrm{W}$. et al. Near-infrared photodetectors based on $\mathrm{MoTe}_{2} /$ graphene heterostructure with high responsivity and flexibility. Small 13, 1-8 (2017).

[30] Wang, F. et al. Strong electrically tunable $\mathrm{MoTe}_{2} /$ graphene van der Waals heterostructures for high-performance electronic and optoelectronic devices. Appl. Phys. Lett. 109, 193111 (2016).

[31] Octon, T. J., Nagareddy, V. K., Russo, S., Craciun, M. F. \& Wright, C. D. Fast high-responsivity few-layer $\mathrm{MoTe}_{2}$ photodetectors. Adv. Opt. Mater. 4, 1750-1754 (2016).

[32] Ruppert, C., Aslan, O. B. \& Heinz, T. F. Optical properties and band gap of single- and few-layer $\mathrm{MoTe}_{2}$ crystals. Nano Lett. 14, 6231-6236 (2014).

[33] Youngblood, N., Chen, C., Koester, S. J. \& Li, M. Waveguide-integrated black phosphorus photodetector with high responsivity and low dark current. Nat. Pho- 
ton. 9, 249-252 (2015).

[34] Desiatov, B., Goykhman, I. \& Levy, U. Demonstration of submicron square-like silicon waveguide using optimized LOCOS process. Opt. Express 18, 18592-18597 (2010).

[35] Naiman, A. et al. Ultrahigh-Q silicon resonators in a planarized local oxidation of silicon platform. Opt. Lett. 40, 1892-1895 (2015).

[36] Zomer, P. J., Guimaraes, M. H. D., Brant, J. C., Tombros, N. \& Van Wees, B. J. Fast pick up technique for high quality heterostructures of bilayer graphene and hexagonal boron nitride. Appl. Phys. Lett. 105, 013101 (2014).

[37] Lee, E. J. H., Balasubramanian, K., Weitz, R. T., Burghard, M. \& Kern, K. Contact and edge effects in graphene devices. Nat. Nanotechnol. 3, 486-490 (2008).

[38] Shin, H.-J. et al. Fermi level pinning at electrical metal contacts of monolayer molybdenum dichalcogenides. ACS Nano 11, 1588-1596 (2017).

[39] Nakaharai, S., Yamamoto, M., Ueno, K. \& Tsukagoshi, K. Carrier polarity control in $\alpha-\mathrm{MoTe}_{2}$ Schottky junctions based on weak Fermi-level pinning. ACS Appl. Mater. Interfaces 8, 14732-14739 (2016).

[40] Wee, A. T. S. et al. Reducing the Schottky barrier between few-layer $\mathrm{MoTe}_{2}$ and gold . 2D Materials 4, 045016 (2017).

[41] Goykhman, I. et al. On-Chip integrated, silicon-graphene plasmonic Schottky photodetector with high responsivity and avalanche photogain. Nano Lett. 16, 3005-3013 (2016).

[42] Mueller, T., Xia, F. \& Avouris, P. Graphene photodetectors for high-speed optical communications. Nat. Photon. 4, 297-301 (2010).

[43] Chui, C., Okyay, A. \& Saraswat, K. Effective dark current suppression with asymmetric msm photodetectors in group iv semiconductors. Photonics Technology Letters, IEEE 15, 1585 - 1587 (2003).

[44] Georgiou, T. et al. Vertical field-effect transistor based on graphene- $\mathrm{WS}_{2}$ heterostructures for flexible and transparent electronics. Nat. Nanotechnol. 8, 100-103 (2013).

[45] Lin, J.-Y. J., Roy, A. M., Nainani, A., Sun, Y. \& Saraswat, K. C. Increase in current density for metal contacts to n-germanium by inserting $\mathrm{TiO}_{2}$ interfacial layer to reduce Schottky barrier height. Appl. Phys. Lett. 98, 092113 (2011).

[46] Zang, H.-J., Kim, G.-S., Park, G.-J., Choi, Y.-S. \& Yu, H.-Y. Asymmetrically contacted germanium photodiode using a metal-interlayer-semiconductor-metal structure for extremely large dark current suppression. Opt. Lett. 41, 3686-3689 (2016).

[47] Massicotte, M. et al. Picosecond photoresponse in van der Waals heterostructures. Nat. Nanotechnol. 11, 42-46 (2015).

[48] He, J. et al. Electron transfer and coupling in graphenetungsten disulfide van der Waals heterostructures. Nat. Comm. 5, 5622 (2014).

[49] Kato, K., Hata, S., Kawano, K. \& Kozen, A. Design of ultrawide-band, high-sensitivity p-i-n photodetectors. IEICE Trans. Electron. E76-C, 214-221 (1993).

[50] Xia, F., Mueller, T., Lin, Y.-M. M., Valdes-Garcia, A. \& Avouris, P. Ultrafast graphene photodetector. Nat. Nanotechnol. 4, 839-843 (2009).
[51] Cui, Q., Ceballos, F., Kumar, N. \& Zhao, H. Transient absorption microscopy of monolayer and bulk $\mathrm{WSe}_{2}$. ACS Nano 8, 2970-2976 (2014).

[52] Shi, H. et al. Exciton dynamics in suspended monolayer and few-layer $\mathrm{MoS}_{2} 2 \mathrm{D}$ crystals. ACS Nano 7, 1072-1080 (2013).

[53] Datta, I. et al. Low-loss composite photonic platform based on $2 \mathrm{~d}$ semiconductor monolayers arXiv:1906.00459. (2019).

[54] Datta, I. et al. Composite photonic platform based on $2 \mathrm{~d}$ semiconductor monolayers. Conference on Lasers and Electro-Optics, FTu3C.2 (2019).

[55] Polat, E. O. et al. Flexible graphene photodetectors for wearable fitness monitoring. Sci. Adv. 5 (2019).

\section{Acknowledgements}

This research was supported by the Swiss National Science Foundation (grant no. 200021_165841). K.W. and T.T. acknowledge support from the Elemental Strategy Initiative conducted by the MEXT, Japan, A3 Foresight by JSPS and the CREST (JPMJCR15F3), JST. This work was carried out partially at the Binnig and Rohrer Nanotechnology Center and the FIRST Center for Micro- and Nanotechnology at ETH Zurich.

\section{Author contributions}

N.F., P.M., J.L. and L.N. conceived the project. N.F and P.M. designed and fabricated the devices and performed the experiments. Y.S. contributed to the experiments. A.E. contributed to the device fabrication. T.T. and K.W. synthesized the hBN crystals. N.F., P.M., J.L. and L.N. analyzed the data and co-wrote the manuscript, with support from all authors.

N.F. and P.M. contributed equally.

\section{Competing Interests}

The authors declare no competing interests.

\section{Additional information}

Supplementary information is available in the online version of the paper. Reprints and permission information is available online at www.nature.com/reprints.

Correspondence and requests for materials should be addressed to P.M.

Reprints and permissions information is available at www.nature.com/reprints. 